\title{
High Curie temperatures in ferromagnetic Cr-doped AIN thin films
}

\author{
D. Kumar \\ Electronic Science Department, Kurukshetra University, Kurukshetra 136 119, India \\ J. Antifakos, M. G. Blamire, ${ }^{\text {a) }}$ and Z. H. Barber \\ Department of Materials Science and Metallurgy, University of Cambridge, Pembroke Street, \\ Cambridge CB2 3QZ, United Kingdom
}

(Received 1 March 2004; accepted 22 April 2004; published online 28 May 2004)

\begin{abstract}
$\mathrm{Al}_{1-x} \mathrm{Cr}_{x} \mathrm{~N}$ thin films with $0.02 \leqslant x \leqslant 0.1$ were deposited by reactive co-sputtering onto $c$-plane (001) sapphire. Room-temperature ferromagnetism with a coercive field of 85 Oe was observed in samples with chromium contents as low as $x=0.027(2.7 \%)$. With increasing $\mathrm{Cr}$ content the mean magnetic moment is strongly suppressed, with a maximum saturation moment of 0.62 and $0.71 \mu_{\mathrm{B}}$ per $\mathrm{Cr}$ atom at 300 and $50 \mathrm{~K}$, respectively. We show that the Curie temperature of $\mathrm{Al}_{1-x} \mathrm{Cr}_{x} \mathrm{~N}$ for $x=0.027$ is greater than 900 K. (C) 2004 American Institute of Physics. [DOI: 10.1063/1.1763216]
\end{abstract}

Since the discovery of carrier-induced ferromagnetism in InMnAs and GaMnAs, ${ }^{1,2}$ there has been rapidly growing interest in the properties and potential applications of dilute magnetic semiconductors (DMSs). The most significant limitation to application of these materials is the low Curie temperature $\left(T_{C}\right)$ generally observed in DMSs based on traditional compound semiconductors. This has prompted many groups to investigate alternatives including magnetically doped oxides such as $\mathrm{ZnO},{ }^{3,4} \mathrm{SnO}_{2},{ }^{5}$ and $\mathrm{TiO}_{2} \cdot{ }^{6}$

Theoretical predictions of room temperature ferromagnetism in $3 d$-transition-metal-doped $\mathrm{GaN}$ indicated that $\mathrm{Mn}$ and $\mathrm{Cr}$-doped $\mathrm{GaN}$ are promising candidate DMSs, with $\mathrm{Cr}$ doped GaN likely to exhibit the most stable ferromagnetic states. ${ }^{7}$ Dietl et al. predicted from a mean field model that Mn-doped GaN should have a $T_{C}$ higher than room temperature. ${ }^{8}$ Based on local-density functional calculations, the exchange interaction between magnetic dopants and III-V compounds may explain the maximum observed critical temperature at different impurity concentrations. ${ }^{9}$

Recently, room temperature ferromagnetism has been experimentally observed in $\mathrm{Mn}$ - and $\mathrm{Cr}$ - doped $\mathrm{GaN}$ and AlN. ${ }^{10-14}$ The $T_{C}$ for $(x=0.03-0.05)$ Mn-doped GaN films has been reported to be over $750 \mathrm{~K}^{15}$ and ferromagnetic behavior with a $T_{C}$ higher than $400 \mathrm{~K}$ has been reported for Cr-doped GaN. ${ }^{16}$ Previous studies on AlN have reported a large variation in the value of mean magnetic moment of Cr-doped AlN films depending on the technique of deposition and doping concentration, ${ }^{13,14}$ but it is clear that high $\mathrm{Cr}$ doping levels $(x \geqslant 0.1)$ result in segregation. To date, samples with varying $\mathrm{Cr}$ composition at levels $(x<0.05)$ have not been studied. In this letter, we report the growth of $\mathrm{Al}_{1-x} \mathrm{Cr}_{x} \mathrm{~N}$ films and show that high $T_{C} \mathrm{~s}$ can be obtained at low doping levels.

Thin film samples of $\mathrm{Al}_{1-x} \mathrm{Cr}_{x} \mathrm{~N}$, were deposited on $c$-plane (001) sapphire substrates by reactive dc cosputtering from an $\mathrm{Al}$ and a $\mathrm{Cr}$ target in an ultrahigh vacuum sputtering chamber surrounded by a liquid-nitrogen filled jacket with a base pressure below $3 \times 10^{-7} \mathrm{~Pa}$. A sputtering

\footnotetext{
a) Author to whom correspondence should be addressed; electronic mail: mb52@cam.ac.uk
}

gas of $70 \% \mathrm{~N}_{2} / 30 \% \mathrm{Ar}$ at a total pressure of $3 \mathrm{~Pa}$ was used. The temperature of the platinum resistive heater, on which the substrates were placed, was held at $940{ }^{\circ} \mathrm{C}$ (using an optical pyrometer); the actual substrate temperature was approximately $150{ }^{\circ} \mathrm{C}$ below this.

The substrate heater was placed between the $\mathrm{Al}$ and $\mathrm{Cr}$ targets such that a series of $\mathrm{Al}_{1-x} \mathrm{Cr}_{x} \mathrm{~N}$ films with different $\mathrm{Cr}$ compositions could be fabricated on a set of $10 \times 5 \mathrm{~mm}^{2}$ substrates in one deposition run. In this geometry $\mathrm{Al}_{1-x} \mathrm{Cr}_{x} \mathrm{~N}$ films with $\operatorname{Cr}(x<0.027)$ could not be deposited because of limitations on the relative powers which could be applied to the $\mathrm{Al}$ and $\mathrm{Cr}$ targets. The concentration of $\mathrm{Cr}$ in the films was estimated by energy dispersive $\mathrm{x}$-ray analysis of films deposited on to silica substrates placed at either end of the heater in each run; previous experiments had shown that our geometry gives an essentially linear composition spread and so the $\mathrm{Cr}$ concentration of each sample could be accurately calculated from its position on the heater. Film structure and crystallographic texture were characterized using a Philips $\mathrm{x}$-ray diffractometer (XRD) equipped with a $\mathrm{Cu}$ source. The magnetic measurements were carried out with the magnetic field parallel to the sample using a Princeton Measurement Corporation vibrating sample magnetometer (VSM) fitted with a furnace and a cryostat which enabled measurements between 10 and $800 \mathrm{~K}$.

The thickness of films varied between $400 \mathrm{~nm}$ and 600 $\mathrm{nm}$. Atomic force microscopy was used to characterize the surface morphology; the mean and root mean square roughness values for a typical film were 4.8 and $6.1 \mathrm{~nm}$, respectively. The surface topography suggested columnar growth.

Our growth conditions resulted in a preferred (002) orientation for pure AlN, i.e., $c$ axis normal to the plane of the film. Figure 1 shows $2 \theta-\theta$ XRD scans of $\mathrm{Al}_{1-x} \mathrm{Cr}_{x} \mathrm{~N}$ thin films with different $\mathrm{Cr}$ levels deposited at the same temperature on $c$-plane sapphire. It can be seen that all the films are predominantly (002) oriented, but that with increasing $x$ the intensities of other reflections increase relative to the (002) diffraction peak. For $x=0.027$ and 0.035 only the (002) reflection at $36.1^{\circ}$ can be observed; for the sample with $x=0.046$ the first indication of the (101) AlN peak appears; and for $x=0.061$ this peak can clearly be observed. The same 


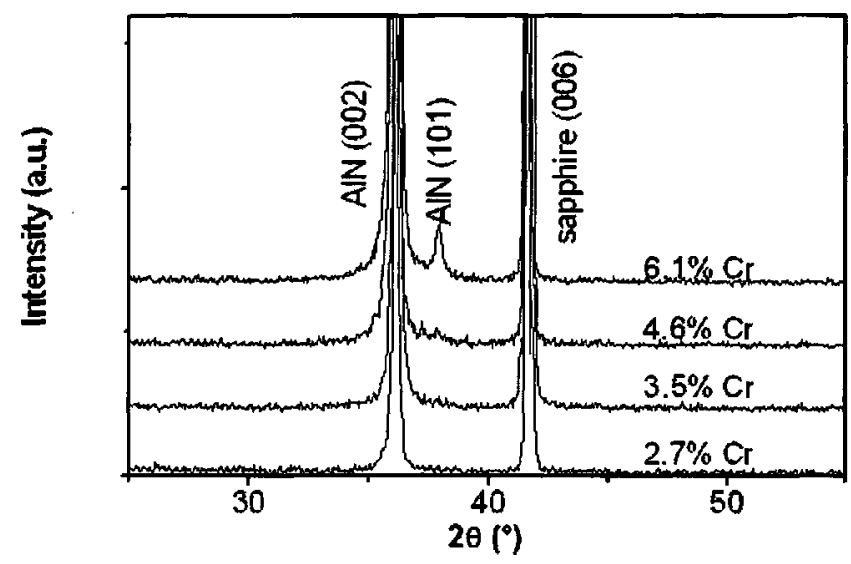

FIG. 1. X-ray diffraction patterns of $\mathrm{Al}_{1-x} \mathrm{Cr}_{x} \mathrm{~N}$ samples with varying $\mathrm{Cr}$ composition deposited on $c$-plane (001) sapphire a heater temperature of $940^{\circ} \mathrm{C}$.

trend was observed in other deposition runs: preferred $c$-axis orientation dropped with increasing $\mathrm{Cr}$ content. No peaks corresponding to any other possible phase, such as $\mathrm{CrN}$, $\mathrm{Cr}_{2} \mathrm{~N}$ or $\mathrm{CrO}_{2}$, were observed.

The magnetization versus magnetic field $(M-H)$ loops for $\mathrm{Al}_{1-x} \mathrm{Cr}_{x} \mathrm{~N}$ with $x=0.027$ and $x=0.061$ at $300 \mathrm{~K}$ are shown in Fig. 2; both loops are strongly hysteretic, but it can be seen that the total moment for $x=0.061$ is substantially lower than for $x=0.027$. $M-H$ loops measured for $x=0.027$ at different temperatures $(20-800 \mathrm{~K})$ were used to calculate the saturation magnetization moment $\left(M_{S}\right)$, magnetic remanent magnetization $\left(M_{R}\right)$ per $\mathrm{Cr}$ atom, as shown in Fig. $3 .^{17}$ Coercive field values as a function of temperature are also shown in this figure. It can be seen that the film is still strongly ferromagnetic at the highest measurement temperature of our system, $800 \mathrm{~K}$.

As expected, the data show a progressive reduction in $M_{S}$ with increasing temperature but, as shown in Fig. 3, we have also consistently observed a reduction in $M_{S}$ values with reducing temperature below than $50 \mathrm{~K}$. The effective magnetic moment per $\mathrm{Cr}$ atom, deduced from $M-H$ loops measured at room temperature, as a function of $x$ is plotted in Fig. 4. The data show a consistent reduction in total moment (inset of Fig. 4) with increasing $\mathrm{Cr}$ concentration.

The major difficulty in making AlN ferromagnetic is the low solubility limit ${ }^{18}$ for magnetic impurities such as $\mathrm{Mn}$ or

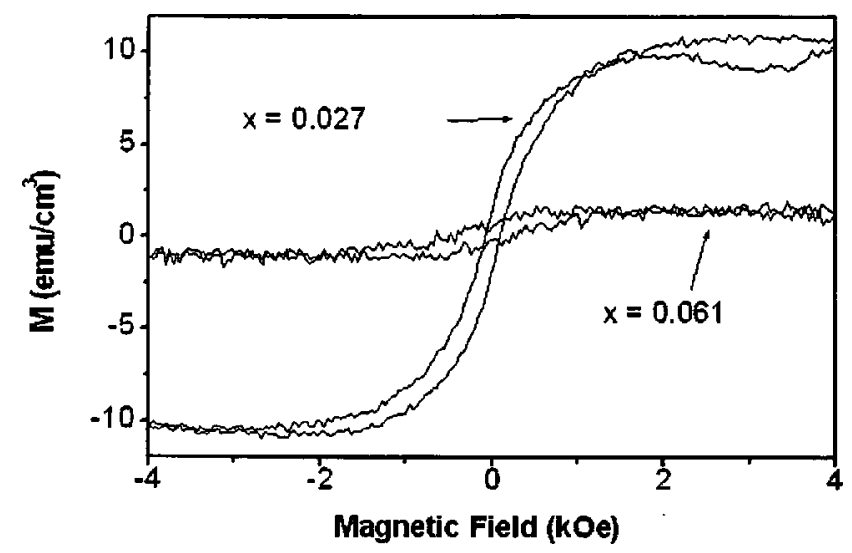

FIG. 2. Magnetic field dependence of the magnetization of $\mathrm{Al}_{1-x} \mathrm{Cr}_{x} \mathrm{~N}$ films for $x=0.027$ and 0.061 at $300 \mathrm{~K}$.

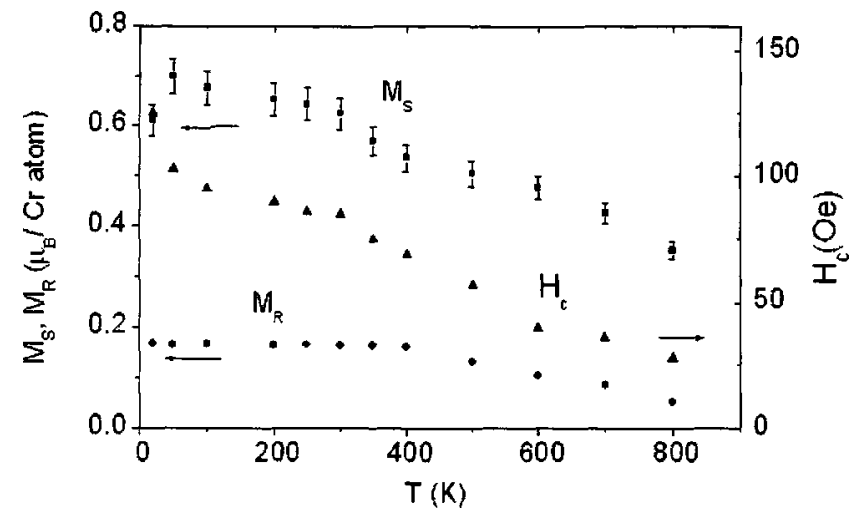

FIG. 3. Temperature dependence of the effective saturation magnetic moment $\left(M_{S}\right)$, magnetic remanent magnetization $\left(M_{R}\right)$, and coercive field $\left(H_{c}\right)$ of $\mathrm{Al}_{1-x} \mathrm{Cr}_{x} \mathrm{~N}$ with $x=0.027$.

Cr. However, there appears to be a narrow processing window in terms of deposition temperature and doping concentration at which effective ferromagnetic doping of AlN is possible and which also avoids second phase formation and maintains the preferred growth orientation. ${ }^{11}$ Our films have been deposited under good vacuum conditions and so ferromagnetic $\mathrm{CrO}_{2}$ formation, possible for the highly doped films of Yang et al., ${ }^{14}$ is extremely unlikely. XRD analysis does not detect any other phase, although this does not necessarily preclude the presence of nano-clusters of $\mathrm{Cr}$ or $\mathrm{Cr}$ compounds.

The remanent magnetization for the $2.7 \%$ doped sample was found to decrease with increasing temperature $(>50 \mathrm{~K})$; however the films were strongly hysteretic at all temperatures. The coercive field and effective magnetization also decreased with increasing temperature. The relatively low signal to noise ratio at high temperatures prevented the use of Arrott plots to determine $T_{C}$; however, the $M-H$ measurements made at higher temperatures clearly indicate that $T_{C}$ is higher than $800 \mathrm{~K}$. Extrapolation of the linear dependence of the remanent magnetization to higher temperatures implies a $T_{C}$ well above $900 \mathrm{~K}$, and probably closer to $1000 \mathrm{~K}$.

The rapid decrease in effective saturation magnetic moment $M_{S}$ per $\mathrm{Cr}$ atom with increasing $\mathrm{Cr}$ content may be associated with the decline in crystal quality with increasing

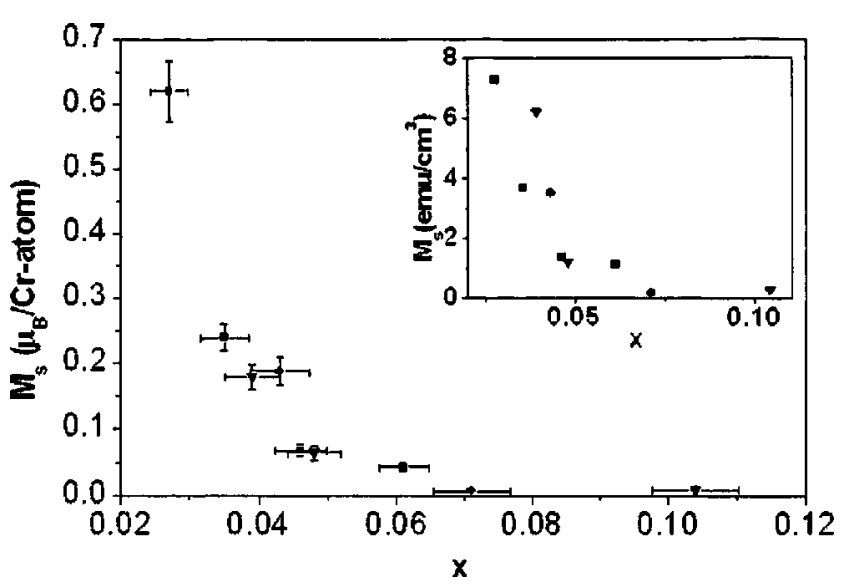

FIG. 4. Effective magnetic moment per $\mathrm{Cr}$ atom for $\mathrm{Al}_{1-x} \mathrm{Cr}_{x} \mathrm{~N}$ film as a function of $\mathrm{Cr}$ concentration measured at $300 \mathrm{~K}$; different growth runs are distinguished by different symbols. The inset shows the same data expressed as moment per unit volume 
$\mathrm{Cr}$ content or may be due to enhanced antiferromagnetic coupling at higher $\mathrm{Cr}$ concentrations. Alternatively, it is possible that this decrease is a consequence of an increasing chemical instability with higher $\mathrm{Cr}$ concentration which may lead to clustering. This decrease in total moment with increasing doping concentration is similar to that already reported for Mn-doped GaN films. ${ }^{19}$ The reason for the decrease in $M_{S}$ $(<50 \mathrm{~K})$ is not understood, but may also indicate a competing antiferromagnetic coupling.

At present, there seems to be no single theoretical model which can explain the ferromagnetism in all DMS materials. Ferromagnetism based on free-carrier mediated models predicted for Mn-doped III-V semiconductors may not be applicable to AlN as it is highly resistive at room temperature. It has recently been proposed that small $\mathrm{Cr}-\mathrm{N}$ clusters could be strongly ferromagnetic, ${ }^{20}$ and so could account for the magnetic moment and high $T_{C}$ s in Cr-doped III-V nitrides. However, this appears to be inconsistent with our data, in which ferromagnetism is strongest at the lowest $\mathrm{Cr}$ concentrations and, as noted by Hori et al. ${ }^{15}$ the small size of the clusters necessary for ferromagnetism seems certain to imply a superparamagnetic behavior which is inconsistent with the hysteresis observed even at the highest temperatures in our films.

In conclusion, we have deposited ferromagnetic $\mathrm{Cr}$ doped AlN films with strong (002) texture on $c$-plane sapphire, showing high saturation magnetization moments of 0.62 and $0.71 \mu_{\mathrm{B}} / \mathrm{Cr}$ atom at 300 and $50 \mathrm{~K}$, respectively, for $\mathrm{Cr}$ contents as low as $2.7 \%$. The magnetization measurements carried out at higher temperatures indicate that the Curie temperature of Cr-doped AlN is higher than $900 \mathrm{~K}$. The most immediate application for high temperature ferromagnetic AlN may be in spin electronic devices as a spinfiltering magnetic tunnel barrier. ${ }^{11}$

D.K. thanks ACU, London for awarding a Commonwealth Fellowship to support this research. The authors thank Dr. Madhav Adyam and Dr. Dennis Leung for their assistance with VSM measurements and for useful discussions.
${ }^{1}$ H. Munekata, H. Ohno, S. von Molnar, A. Segmuller, L. L. Chang, and L. Esaki, Phys. Rev. Lett. 63, 1849 (1989).

${ }^{2}$ H. Ohno, A. Shen, F. Matsukura, A. Oiwa, A. Endo, S. Katsumoto, and Y. Iye, Appl. Phys. Lett. 69, 363 (1996).

${ }^{3}$ K. Sato and H. Katayama-Yoshida, Jpn. J. Appl. Phys., Part 2 40, L334 (2001).

${ }^{4}$ H. J. Lee, S. Y. Jeong, C. R. Cho, and C. H. Park, Appl. Phys. Lett. 81, 4020 (2002).

${ }^{5}$ S. B. Ogale, R. J. Choudhary, J. P. Buban, S. E. Lofland, S. R. Shinde, S. N. Kale, V. N. Kulkarni, J. Higgins, C. Lanci, J. R. Simpson, N. D. Browning, S. Das Sarma, H. D. Drew, R. L. Greene, and T. Venkatesan, Phys. Rev. Lett. 91, 077205 (2003).

${ }^{6}$ W. K. Park, R. J. Ortega-Hertogs, J. S. Moodera, A. Punnoose, and M. S. Seehra, J. Appl. Phys. 91, 8093 (2002).

${ }^{7}$ K. Sato and H. Katayama-Yoshida, Jpn. J. Appl. Phys., Part 2 40, L485 (2001).

${ }^{8}$ T. Dietl, H. Ohno, F. Matsukura, J. Cibert, and D. Ferrand, Science 287, 1019 (2000).

${ }^{9}$ M. van Schilfgaarde and O. N. Mryasov, Phys. Rev. B 63, 233205 (2001).

${ }^{10}$ M. L. Reed, N. A. El-Masry, H. H. Stadelmaier, M. K. Ritums, M. J. Reed, C. A. Parker, J. C. Roberts, and S. M. Bedair, Appl. Phys. Lett. 79, 3473 (2001).

${ }^{11}$ R. Frazier, G. Thaler, M. Overberg, B. Gila, C. R. Abernathy, S. J. Pearton, and N. Newman, Appl. Phys. Lett. 82, 3047 (2003).

${ }^{12}$ S. E. Park, H.-J. Lee, Y. C. Cho, S.-Y. Jeong, C. R. Cho, and S. Cho, Appl. Phys. Lett. 80, 4187 (2002).

${ }^{13}$ S. Y. Wu, H. X. Liu, L. Gu, R. K. Singh, L. Budd, M. van Schilfgaarde, M. R. McCartney, D. J. Smith, and N. Newman, Appl. Phys. Lett. 82, 3047 (2003).

${ }^{14}$ S. G. Yang, A. B. Pakhomov, S. T. Hung, and C. Y. Wong, Appl. Phys. Lett. 81, 2418 (2002).

${ }^{15}$ H. Hori, S. Sonada, T. Sasaki, Y. Yamamoto, S. Shimizu, K. Suga, and K. Kindo, Physica B 324, 142 (2002).

${ }^{16}$ M. Hashimoto, Y. Zhou, M. Kanamura, and H. Asahi, Solid State Commun. 122, 37 (2002).

${ }^{17}$ The magnetization moment values measured at high temperature $\left(>400{ }^{\circ} \mathrm{C}\right)$ using VSM in furnace mode required a different sample geometry and so these data have been scaled to the value obtained at room temperature.

${ }^{18}$ S. A. Chambers and Y. K. Yoo, MRS Bull. 28, 706 (2003).

${ }^{19}$ G. T. Thaler, M. E. Overberg, B. Gila, R. Frazier, C. R. Abermathy, S. J. Pearton, J. S. Lee, S. Y. Lee, Y. D. Park, Z. G. Khim, J. Kim, and F. Ren, Appl. Phys. Lett. 80, 3964 (2002).

${ }^{20}$ Q. Wang, Q. Sun, B. K. Rao, P. Jena, and Y. Kawazoe, J. Chem. Phys. 119, 7124 (2003) 